Discussion Paper No. 551

\title{
HETEROGENEOUS HABITS AND \\ THE TRANSFER PARADOX
}

\author{
Ichiro Gombi \\ and \\ Shinsuke Ikeda
}

\begin{abstract}
August 2001
The Institute of Social and Economic Research

Osaka University

6-1 Mihogaoka, Ibaraki, Osaka 567-0047, Japan
\end{abstract}




\title{
Heterogeneous Habits and The Transfer Paradox
}

\author{
Ichiro Gombi* and Shinsuke Ikeda $\dagger \ddagger$ \\ Ritsumeikan University and Osaka University
}

August 2001

By using a two-country model with habit-forming consumers, this paper shows that the transfer paradox can take place in the free-trade, dynamically-stable world economy. When the debtor is more habituated to consumption than the creditor, an income transfer from the creditor to the debtor raises the interest rate in transition through changes in time preference. With sufficiently low elasticities of intertemporal substitution and/or sufficiently large stock of the creditor's assets, the intertemporal terms of trade effect immiserizes the recipient and enriches the donor. Although the transfer paradox occurs only when the international bond market is "unstable" with respect to an ad hoc Walrasian adjustment process, the equilibrium dynamics are stable in the usual sense: given that the economy is always in the rational expectation equilibrium, the transfer paradox generically occurs.

JEL Classification Numbers: F00, D90.

Keywords: Habit, transfer paradox, two-country model, stability.

*I. Gombi, The Faculty of Economics, Ritsumeikan University, Kusatsu, Shiga 525-8577 Japan. Telephone: 81-77-561-4840, Email: <gombi@ec.ritsumei.ac.jp>.

${ }^{\dagger}$ Corresponding author: S. Ikeda, The Institute of Social and Economic Research, Osaka University, Mihogaoka, Ibaraki, Osaka 567-0047, Japan. Telephone: 81-6-68798568, Facsimile: 81-6-6878-2766, Email: <ikeda@iser.osaka-u.ac.jp>.

${ }^{\ddagger}$ We are very grateful to F. Ohtake, R. Okumura, Y. Ono, S. Takagi, and the participants at the JEA Spring Meeting, Hiroshima Shudo University, and the Macro Research Seminar for helpful comments and discussions on earlier versions of the paper. 


\section{Introduction}

Since Professor Paul Samuelson showed in his seminal work (Samuelson $(1947,1952))$ that the transfer paradox, i.e., income transfers which enrich donors and immiserize recipients, cannot take place in two-country Walrasstable equilibria insofar as there is no distortion, it has been the fundamental result in the literature of the transfer problem. Starting from the result, the trade theorists have made considerable contributions by (i) adding the third country and/or by (ii) incorporating distortions. ${ }^{1}$

The contributions, however, have been limited to intrinsically static analysis and, except for several articles cited later, the intertemporal aspects of the transfer problem have not been extensively examined. With international financial markets recently getting more and more integrated, international income transfers may well induce variations in interest rates and in asset prices, thereby bringing about rapid and huge endogenous re-transfers of resources. To incorporate this effect using a dynamic-optimizing model would help obtain a new insight into the transfer problem.

The purpose of this paper is to examine the transfer problem in a twocountry world economy with infinitely-lived, heterogeneously habit-forming consumers. Particularly emphasized is international heterogeneity in habituation to consumption. With the resultant heterogeneity in intertemporal consumption complementarities, income transfers instantaneously affect the world interest rate, thereby causing the intertemporal terms of trade effects on both of the countries. By incorporating the effects, we show that, without any distortion, the dynamically-stable equilibrium of the two-country world economy allows income transfers to immiserize the recipient and enrich the donor.

As a stylized fact, habit formation has been often reported as playing important roles in determining actual consumer behavior (e.g., Braun, Constantinides, and Ferson (1993), Naik and Moore (1996), Campbell and Cochrane (1999), Carrol, Overland, and Weil (2000), and Boldrin, Lawrence, and Fisher (2001)). However, without no exception, the existing models of con-

\footnotetext{
${ }^{1}$ For the literature of (i), see, e.g., Gale (1974), Yano (1983), and Bhagwati, Brecher, and Hatta (1983). The literature of (ii) contains Brecher and Bhagwati (1982), Bhagwati, Brecher, and Hatta (1985), and Yano and Nugent (1999). To be precise, the presence of the third country can be regarded as a distortion insofar as the transferer and the transferee do not jointly levy the optimal tariffs against the third country. For this point, see Bhagwati, Brecher, and Hatta (1983).
} 
sumption habits commonly rule out consumers' interactions due to pecuniary externalities: they focus on representative agent models (e.g., Ryder and Heal (1973) and Carrol et al. (2000)) and/or small country models (Mansoorian (1993) and Ikeda and Gombi (1999)). When the interest rate is endogenously determined in heterogeneous consumer (or country) economies, one's consumption-habit behavior would be affected by others' through interestrate adjustments.

To characterize such internationally interactive habit dynamics, we construct a world felicity function from the two countries' individual felicity functions. It captures pecuniary externalities caused by interest rate adjustments helps describe the two-country equilibrium easily. Using the approach, the possibility of the transfer paradox is shown to depend on: (i) international differences in the degree of habituation; (ii) initial asset distribution; and (iii) elasticities of intertemporal substitution. In particular, when the debtor is more habituated to consumption than the creditor, an income transfer from the creditor to the debtor raises the interest rate in transition through changes in time preference. With sufficiently low elasticities of intertemporal substitution and/or sufficiently large stock of the creditor's assets, the resultant intertemporal terms of trade effect immiserizes the recipient and enriches the donor.

A punch-line of the result is that it is seemingly inconsistent with Samuelson's fundamental result: he claimed that the paradox never occurs in twocountry, free-trade Walras-stable equilibria. To reconcile the two propositions, we introduce an ad hoc Walras adjustment process, showing that our transfer paradox also takes place only when the international bond market is "unstable" with respect to the ad hoc process. By doing it, however, we do not intend to claim that the transfer paradox is implausible: the adjustment process is not a rational-expectation equilibrium. The rationalexpectation equilibrium we obtain is dynamically (saddle-point) stable in the usual sense. Our point is: given that the economy is always in the dynamic rational expectation equilibrium, the transfer paradox generically occurs at the neighborhoods of a certain steady state point.

\subsection{Comparisons with the literature}

There are several important contributions in the dynamic theory of the transfer problem. Among others Epstein (1987) is related most closely to the present research. In an application of his dynamic general equilibrium model 
with recursive preferences, he predicts that the transfer paradox can occur under "turnpike stability" (Epstein (1987), footnote 8, p.91). In his model, time preference, as a source of interest-rate adjustments, is endogenized by formalizing directly the discount rate as a function of consumption. In our non-recursive preference model, variations in time preference are generated endogenously by habit formation, in contrast. Furthermore, he does not examine how his result can be reconciled to Samuelson's result. ${ }^{2}$

Fries (1983) gives an example of the transfer paradox in a two-country, two-commodity trade model with production uncertainty. The example is constructed by assuming that equities are internationally-nontraded. On the contrary, we emphasize the possibility that rapid interest-rate adjustments in well-organized international capital markets bring about paradoxical intertemporal terms-of-trade effects.

Galor and Polemarchakis (1987) examine the possibility of the transfer paradox in the overlapping-generation framework. In their paper, dynamic inefficiency arising from the overlapping-generation structure plays a critical role in producing the paradox. The analysis is, however, limited to the steady-state effect on welfare, neglecting utility gains and losses on the transition path. We consider efficient equilibria in an infinitely-lived agent model, where welfare effects are evaluated by taking into account the entire time-profile of consumption. The punch-line lies in the finding that without allocative inefficiency, the intertemporal terms of trade effect due to international heterogeneity in habits can cause the paradox.

Djajić, Lahiri, and Raimondos-Møller (1998) examine the intertemporal terms of trade effect of income transfers by using a two-period model. Although our attempt is similar to theirs in spirits, they rule out the possibility of the transfer paradox by adopting a time-additive utility function. We incorporate time-nonseparability of preferences in the form of consumption habits, which has two effects: First, it brings intrinsic dynamics into the model. Second, it enables us to endogenize transitional shifting of time preference, the dynamics of the interest rate, and the intertemporal terms of trade effect. ${ }^{3}$

\footnotetext{
${ }^{2}$ As a minor difference, in his model the interest rate can adjust only gradually over time as the aggregate capital stock varies. In our model, in contrast, a transfer instantly affects the interest rate through the market clearing condition of the "present-good" market.

${ }^{3}$ After we completed an earlier version of the paper, Professor S. Lahiri kindly sent us the manuscript of Djajić, Lahiri, and Raimondos-Møller (2001). It focuses on consumption habits through which a temporary foreign aid affects the terms of trade and welfare of
} 
Our discussion is also related to the literature on the "correspondence principle" initially proposed by Samuelson (1947): By imposing the stability conditions on "disequilibrium" dynamics, one can derive "fruitful" results in comparative statics. The trade theorists including Samuelson himself applied this principle to rule out the transfer paradox. As pointed out in a critical survey by Kemp (1987), however, with re-emergence of dynamic theory of macroeconomics, economists have sought to consider (saddle-point) stability of intrinsically dynamic stock-flow processes by assuming that the economy is always in dynamic equilibrium. For example, Burmeister and Long (1977) reasonably proposed a new correspondence principle relating the saddle-point stability and steady-state properties in dynamic optimization models, showing that some paradoxes can easily survive under the new criterion. Our result also implies that the transfer paradox cannot be ruled out by the new correspondence principle.

The rest of the paper proceeds as follows: In Section 2, we present a twocountry model with consumption habits. In Section 3, equilibrium dynamics are derived and characterized. In Section 4, the effects of transfer payments are examined, where the possibility of the transfer paradox is shown in the main proposition. Section 5 discusses the possibilities of immiserizing transfers more explicitly by considering quadratic felicity functions. Section 6 summarizes the paper with concluding remarks.

\section{The Two-Country Model}

Consider a two-country world economy composed of home and foreign countries. Each country is populated with infinitely-lived identical agents. The representative agents in home and foreign countries are referred to as consumers $\mathrm{H}$ and $\mathrm{F}$, respectively. They consume a single consumption good and hold wealth in the form of bonds. Both the goods and bonds are traded freely in international markets. For brevity, the representative agents $\mathrm{H}$ and $\mathrm{F}$ are assumed to be endowed with constant amounts of output $y$ and $y^{*}$, respectively. Throughout the paper, foreign country's variables are represented with superscript asterisks.

Consumption forms habits. Letting $z_{t}^{(*)}$ represent the time- $t$ habit, we

the donor and recipient countries. In contrast to our setting, they introduce distortion by assuming that there is no international asset trade; assume that habits are externalities; and consider a two-period model. 
specify $z_{t}^{(*)}$ as the average of past consumption rates $c_{s}^{(*)}, s \leq t$ : $z_{t}^{(*)}=$ $\alpha \int_{-\infty}^{t} c_{s}^{(*)} \exp (-\alpha(t-s)) d s$, or equivalently

$$
\dot{z}_{t}^{(*)}=\alpha\left(c_{t}^{(*)}-z_{t}^{(*)}\right)
$$

where $\dot{x}$ represents the time derivative of variable $x$; and $\alpha$ represents the discount rate for past consumption rates. We assume that consumers in both of the countries have the common discount rate $\alpha$ for past consumption rates. This enables us to have tractable dynamics of two-country equilibrium.

Consumers $\mathrm{H}$ and $\mathrm{F}$ have different preferences over consumption and habits. Consumer H's preferences are specified as

$$
U_{0}=\int_{0}^{\infty} u\left(c_{t}, z_{t}\right) \exp (-\theta t) \mathrm{d} t
$$

where $\theta$ represents the subjective discount rate, which is assumed constant. Following Ryder and Heal (1973), function $u$ is assumed to satisfy the following regularity conditions: $(\mathrm{C} 1) u_{c}>0 ;(\mathrm{C} 2) u_{z} \leq 0$; (C3) $u_{c}(c, c)+u_{z}(c, c)>$ 0 ; (C4) $u$ is concave in $(c, z)$; (C5) $\lim _{c \rightarrow 0} u_{c}(c, z)=\infty$ uniformly in $z$; and (C6) $\lim _{c \rightarrow 0}\left[u_{c}(c, c)+u_{z}(c, c)\right]=\infty$. Consumer F's utility $U_{0}^{*}$ is specified in the same way.

Due to habit formation, consumer preferences are intertemporally dependent. Within the single-agent framework, Ryder and Heal (1973) characterize the resulting intertemporal complementarities in consumption by adjacent and distant complementarities: With adjacent complementarity, an increase in today's habits increases the marginal utility of today's consumption more than it increases marginal disutility of habits, thereby enlarging the today's optimal consumption. Under distant complementarity, an increase in today's habits increases marginal disutility of habits so much that it reduces today's consumption rate. We apply their idea to characterize each country's subjective or local preferences as follows:

Definition: Intertemporal consumptions in country $\mathrm{H}$ are said to locally or subjectively display adjacent (distant) complementarity, or simply display local or subjective adjacent (distant) complementarity, LAC (LDC), if and only if (iff) $u_{c z}(c, c)+\frac{\alpha}{\theta+2 \alpha} u_{z z}(c, c)>(<) 0$. Similarly LAC and LDC for country $\mathrm{F}$ are defined by using $u^{*}{ }^{4}$

\footnotetext{
${ }^{4}$ Note that we are using term "local" for the meaning of "regional" or "country-specific", not the mathematical meaning of "neighborhoods". Likewise we will later use "global" for the geographical meaning.
} 
In the case of the single agent economy, these subjective complementarities definitely determine the equilibrium dynamics, as seen from the existing literature (e.g., Ryder and Heal (1973) and Ikeda and Gombi (1999)). In contrast, when the economy consists of heterogeneous agents as in the present case, some pecuniary externalities through markets divert their consumptionsaving behavior from what is predicted from the subjective intertemporal complementarities, as shown in the next section.

To ensure the steady state, let us assume that

$$
\theta=\theta^{*} \text {. }
$$

Unlike in the single agent model, however, the interest rate $r$ can deviate from this subjective discount rate.

Let $b_{t}$ denote net foreign assets held by country $\mathrm{H}$. Given the initial values $\left(b_{0}, z_{0}\right)$, consumer $\mathrm{H}$ chooses $C_{0}=\left\{c_{t}, b_{t}, z_{t}\right\}_{t=0}^{\infty}$ so as to maximize (2) subject to: (i) the flow budget constraint,

$$
\dot{b}_{t}=r_{t} b_{t}+y-c_{t}-\tau_{t},
$$

where $\tau_{t}$ denotes lump-sum transfer payments from country $\mathrm{H}$ to $\mathrm{F}^{5}{ }^{5}$ (ii) the formation of consumption habits (1); and (iii) the transversality conditions.

Letting $\lambda_{t}(\geq 0)$ be the shadow price of savings and $\xi_{t}(\leq 0)$ that of habit formation, the optimal conditions are given by

$$
\begin{aligned}
& u_{c}(c, z)=\lambda_{t}-\alpha \xi_{t}, \\
& \dot{\lambda}_{t}=\left(\theta-r_{t}\right) \lambda_{t}, \\
& \dot{\xi}_{t}=(\theta+\alpha) \xi_{t}-u_{z}\left(c_{t}, z_{t}\right),
\end{aligned}
$$

together with (1), (3), and the transversality conditions for $b_{t}$ and $z_{t}$.

In exactly the same way, country F's behavior can be specified. The model is closed by introducing the market clearing conditions:

$$
\begin{aligned}
& c_{t}+c_{t}^{*}=Y\left(\equiv y+y^{*}\right), \\
& b_{t}+b_{t}^{*}=0 .
\end{aligned}
$$

By the Walras law, these are not independent: (8) together with (3) and the corresponding constraint for the foreign consumer imply (7). In sum, the equilibrium time-path of $\left(b_{t}, b_{t}^{*}, c_{t}, c_{t}^{*}, z_{t}, z_{t}^{*}, r_{t}, \lambda_{t}, \lambda_{t}^{*}, \xi_{t}, \xi_{t}^{*}\right)$ is determined by equations (1), (4) through (8), and the transversality conditions for $\left(b_{t}, b_{t}^{*}, z_{t}, z_{t}^{*}\right)$.

\footnotetext{
${ }^{5}$ Without loss of generality, we assume away the presence of governments in the both
} countries since this paper just focuses on the effects of international transfer payments. 


\section{Equilibrium Dynamics}

\subsection{The world felicity function}

In this economy, consumption dynamics in the two countries interact through international bond markets. To ease the analysis, it is useful to introduce "aggregate habits" $Z_{t}$ as

$$
Z_{t} \equiv z_{t}+z_{t}^{*}
$$

Since $\alpha$ is assumed to be internationally identical, the dynamics of $Z_{t}$ can be expressed from (1) and the market clearing condition (7) as $\dot{Z}_{t}=\alpha\left(c_{t}+c_{t}^{*}-Z_{t}\right)=$ $\alpha\left(Y-Z_{t}\right)$. Since $Y$ is constant throughout the paper, this can be solved as

$$
Z_{t}=Y \text {. }
$$

We can thus express $z_{t}^{*}$ as a function of $z_{t}:^{6}$

$$
z_{t}^{*}=Y-z_{t} .
$$

By using (9), dynamics can be drastically reduced in the following manner. Define $\sigma$ as:

$$
\sigma=\frac{\lambda}{\lambda^{*}}
$$

which is constant since $\dot{\lambda}_{t} / \lambda_{t}=\dot{\lambda}_{t}^{*} / \lambda_{t}^{*}$ from (5). We then construct aggregate indices for $\left(u, u^{*}\right)$ and $\left(\xi, \xi^{*}\right)$ as:

$$
\begin{aligned}
& v(c, z) \equiv u(c, z)+\sigma u^{*}(Y-c, Y-z), \\
& \varsigma \equiv \xi-\sigma \xi^{*},
\end{aligned}
$$

where (7) and (9) are substituted.

The new felicity function $v$ represents the aggregate utility function with weights being given by relative individual marginal utilities. We could call it the world felicity function. The new shadow price $\varsigma$ is the weighted difference in the shadow prices of habits. From the definition of $v, \varsigma$ could be regarded as net marginal gains of transferring consumption capital from country $\mathrm{F}$

${ }^{6}$ When aggregate consumable income $Y$ varies due to fiscal policies and/or supply shocks, $Z_{t}$ evolves over time. For this case, see our companion paper, Ikeda and Gombi (2001). 
to $\mathrm{H}$. With these definitions, we can reduce the equilibrium dynamics of consumption habits around a steady state point as follows:

Property 1: In equilibrium, consumer H's habits around steady state are governed by

$$
\begin{aligned}
& \left(\begin{array}{c}
\dot{z} \\
\dot{\varsigma}
\end{array}\right)=M\left(\begin{array}{c}
\hat{z} \\
\hat{\varsigma}
\end{array}\right) ; \\
& M=\left(\begin{array}{ll}
-\alpha\left(1+\frac{v_{c z}}{v_{c c}}\right) & -\frac{\alpha^{2}}{v_{c c}} \\
\frac{\left(v_{c z}\right)^{2}-v_{c c} v_{z z}}{v_{c c}} & \theta+\frac{\alpha\left(v_{c c}+v_{c z}\right)}{v_{c c}}
\end{array}\right),
\end{aligned}
$$

where $\hat{x}$ denotes deviations of variable $x$ from its steady-state value $\bar{x}: \hat{x} \equiv$ $x_{t}-\bar{x}$.

\section{Proof. See Appendix A.}

This property can be easily understood by recalling the second theorem of public economics. Without any distortion, the Pareto-optimal resource allocation attained in this equilibrium can be duplicated as a solution to a social welfare maximization problem: ${ }^{7}$

$$
\begin{aligned}
& \max U_{0}+\beta U_{0}^{*} \\
& \text { subject to } Y=c_{t}+c_{t}^{*}, Y=z_{t}+z_{t}^{*}, \text { and } \dot{z}_{t}=\alpha\left(c_{t}-z_{t}\right) .
\end{aligned}
$$

Indeed, the corresponding Hamiltonian function,

$$
H_{t}=u\left(c_{t}, z_{t}\right)+\beta u^{*}\left(Y-c_{t}, Y-z_{t}\right)+\varsigma_{t} \alpha\left(c_{t}-z_{t}\right),
$$

produces the same optimal condition as in Property 1 when weight $\beta$ is set equal to $\sigma$.

The dynamic equation (12) for habits is exactly the same as in the case of the small country model (e.g., Ikeda and Gombi (1999) and Mansoorian (1996)), except for that $v$ and $\varsigma$ represent the world average preferences, instead of individual consumers' preferences. In parallel with intertemporal complementarities with respect to consumers' individual preferences, we coin terms to characterize those with respect to the world felicity function:

${ }^{7}$ The Pareto-optimality is shown in footnote 10. 
Definition: Intertemporal consumptions are said to globally display distant (adjacent) complementarity, or simply display global adjacent (distant) complementarity, GAC (GDC), iff $v_{c z}(c, c)+\frac{\alpha}{\theta+2 \alpha} v_{z z}(c, c)>(<) 0$.

From equation (12), we can obtain the characteristic equation: $F(\varpi)=0$, where

$$
F(\varpi) \equiv \varpi^{2}-\theta \varpi-\alpha(\theta+\alpha)-\frac{\alpha(\theta+2 \alpha)}{v_{c c}}\left(v_{c z}+\frac{\alpha}{\theta+2 \alpha} v_{z z}\right),
$$

which has one stable and one unstable roots. Let $\omega$ be the stable root, which is given in terms of the world felicity function as

$$
\omega \equiv \frac{\theta-\sqrt{(\theta+2 \alpha)^{2}-4 \alpha(\theta+2 \alpha) \Omega}}{2}(<0),
$$

where $\Omega \equiv-\left(v_{c z}+\frac{\alpha}{\theta+2 \alpha} v_{z z}\right) / v_{c c}>$ (resp. $\left.<\right) 0$ iff GAC (resp. GDC) prevails.

This root specifies a saddle trajectory for the equilibrium consumption dynamics. In particular, since $\hat{z}_{t}=\omega \hat{z}_{t}$ on the trajectory, the $\hat{c}-\hat{z}$ stable arm is obtained by substituting it into (1) as

$$
\hat{c}_{t}=\left(\frac{\omega+\alpha}{\alpha}\right) \hat{z}_{t}
$$

which, from the market clearing condition and (9), implies:

$$
\hat{c}_{t}^{*}=\left(\frac{\omega+\alpha}{\alpha}\right) \hat{z}_{t}^{*}
$$

From equations (14) through (16), in the case of GAC $\left(v_{c z}(c, c)+\frac{\alpha}{\theta+2 \alpha} v_{z z}(c, c)>\right.$ 0 ), $\omega+\alpha$ is negative and thus the stable arms (15) and (16) are positively sloping, whereas under GDC $\left(v_{c z}(c, c)+\frac{\alpha}{\theta+2 \alpha} v_{z z}(c, c)<0\right)$ the trajectories have a negative slope with negative $\omega+\alpha$. These properties can be summarized as follows:

Proposition 1: In equilibrium, both of the countries experience positive (negative) co-movements between consumption and habits, i.e., $d c_{t}^{(*)} / d z_{t}^{(*)}>$ $(<) 0$, iff the world felicity function displays $G A C(G D C)$.

This proposition implies that the equilibrium dynamics in each country can differ from what is predicted by local habit preferences in individual 
countries: For example, even if consumers in country $\mathrm{H}$ have the LDC preference, their consumption positively co-moves with habits in equilibrium if the world felicity function displays GAC. Note, however, that, for this to be the case, country $\mathrm{F}$ should display strong LAC since the world preference captured by the world felicity function is an average of the two countries' local preferences. More explicitly, by introducing indices $\Omega^{H}$ and $\Omega^{F}$ for local preferences as

$$
\Omega^{H}=-\left(u_{c z}+\frac{\alpha}{\theta+2 \alpha} u_{z z}\right) / u_{c c} \text { and } \Omega^{F}=-\left(u_{c z}^{*}+\frac{\alpha}{\theta+2 \alpha} u_{z z}^{*}\right) / u_{c c}^{*}
$$

where, e.g., $\Omega^{H}>(<) 0 \Leftrightarrow$ LAC (LDC) for H, subjective intertemporal complementarities in individual countries can be related to intertemporal complementarity prevailing globally in equilibrium as follows:

Property 2: Global intertemporal complementarity is a weighted average of the individual countries' local intertemporal complementarities in the sense that:

$$
\Omega=\epsilon \Omega^{H}+(1-\epsilon) \Omega^{F}
$$

where: $\epsilon=u_{c c} /\left(u_{c c}+\sigma u_{c c}^{*}\right)$.

Therefore, LAC (LDC) in both countries implies GAC (GDC). When the local intertemporal complementarities differ internationally, global intertemporal complementarity is determined by their relative strength.

The exact meaning of the local complementarities can be explained as follows. Define characteristic polynomial function $F^{H}(\varpi)$ by replacing the second order derivatives $v_{c c}, v_{c z}$, and $v_{z z}$ in (13) with $u_{c c}, u_{c z}$, and $u_{z z}$, respectively. $F^{H}(\varpi)$ is a characteristic polynomial function for country $\mathrm{H}$ when $r$ is fixed to $\theta$ as if country $\mathrm{H}$ were a small country. The characteristic equation $F^{H}(\varpi)=0$ has again one stable and one unstable roots. When $\omega^{H}$ represents the stable root, it is given in parallel with (14) as

$$
\omega^{H} \equiv \frac{\theta-\sqrt{(\theta+2 \alpha)^{2}-4 \alpha(\theta+2 \alpha) \Omega^{H}}}{2}(<0),
$$

implying that the sign of $\omega^{H}+\alpha$ corresponds to local intertemporal complementarities in country $H$. The same discussion can be applied to the 
case of country $\mathrm{F}$ by introducing the corresponding characteristic equation $F^{F}(\varpi)=0$ and its stable root $\omega^{F}$. Local intertemporal complementarities can be characterized as follows:

\section{Property 3:}

$$
\omega^{i}+\alpha>(<) 0 \Leftrightarrow \text { LAC (LDC) in country } i(i=H, F) .
$$

From the definitions of $\omega, \omega^{H}$, and $\omega^{F}, \omega$ lies between $\omega^{H}$ and $\omega^{F}$. Root $\omega^{H}$ can be either larger or smaller than $\omega^{F}$, depending on whether $\Omega^{H}$ is larger or smaller than $\Omega^{F}$. When $\omega^{H}$ is larger than $\omega^{F}$, consumption adjustment in country $\mathrm{H}$ is slower due to stronger habituation to current consumption than in country F. We describe this relationship by using the following terminology:

Definition: Country $\mathrm{H}$ is said to be more (less) habituated to consumption than country $\mathrm{F}$ iff $\omega^{H}>(<) \omega^{F}$ and hence iff $\Omega^{H}>(<) \Omega^{F}$.

\subsection{Interest rate dynamics and pecuniary externalities}

As shown in Proposition 1, equilibrium consumption dynamics are determined by globally formed habit preferences, which generally differ from local preferences characterized by Property 3. This discrepancy is caused by pecuniary externalities of interest-rate adjustments, as shown in the next subsection. The interest-rate dynamics play an important role in global habit formation and consumption behavior in each country. As is proven by Appendix $\mathrm{B}$, the interest rate dynamics can be described by:

$$
\hat{r}=\kappa\left(\Omega^{H}-\Omega^{F}\right) \hat{z},
$$

where $\kappa$ is defined as

$$
\kappa \equiv \frac{\omega u_{c c} u_{c c}^{*}(\theta+2 \alpha)}{\lambda^{*}(\alpha+\theta-\omega)\left(u_{c c}+\sigma u_{c c}^{*}\right)}(>0) .
$$

This reveals that how the interest rate co-moves with habits in country $\mathrm{H}$ depends crucially on the signs of difference $\Omega^{H}-\Omega^{F}$, and hence on whether country $\mathrm{H}$ is more or less habituated to consumption than country $\mathrm{F}$ : 
Property 4: The equilibrium interest rate positively (negatively) co-moves with country H's habits iff consumption in country $\mathrm{H}$ is more (less) habituated to consumption than in country $\mathrm{F}$ : in transition, we have

$$
\frac{r(t)-\theta}{z(t)-\bar{z}} \gtreqless 0 \Leftrightarrow \omega^{H} \gtreqless \omega^{F} \text { (i.e., } \Omega^{H} \gtreqless \Omega^{F} \text { ). }
$$

Remark: If country $\mathrm{H}$ is identical to country $\mathrm{F}\left(\omega^{H}=\omega^{F}\right)$, or if country $\mathrm{F}$ is small in the sense that $\lambda^{*}$ is infinitely large $\left(\lambda^{*} \rightarrow \infty\right)$, for which case $\kappa$ is zero, the interest rate dynamics degenerate at $\theta$ as in the small country case (e.g., Ikeda and Gombi (1999))

In the presence of international heterogeneity in the habit preference, the interest rate $r(t)$ in transition can deviate from $\theta$. For example, suppose that country H's preferences display LDC $\left(\omega^{H}+\alpha<0\right)$ whereas country F LAC $\left(\omega^{F}+\alpha>0\right)$. Then, $\omega^{F}$ is larger than $\omega^{H}$, so that $r(t)$ negatively comoves with $z(t)$. This can be explained intuitively as follows. Without loss of generality, suppose that $z(t)<\bar{z}$, and hence, from $(9), z^{*}(t)>\bar{z}^{*}$. With LDC, country $\mathrm{H}$ would consume more now than in the future steady state if $r$ equaled $\theta$. Country $\mathrm{F}$ would also consume more now than in the future due to LAC, which would give rise to excess demand in the "present good" market. The market clearing $r(t)$ should thus be higher than its steady state level $\theta$, as shown in Figure 1. By the same reasoning, in the case of LAC for country $\mathrm{H}$ with LDC prevailing in country $\mathrm{F}, r(t)$ is positively correlated with $z(t)$.

These interest rate adjustments cause pecuniary externalities that divert consumption dynamics from what is predicted from individual countries' preferences, as pointed out in Proposition 1. Recall, for example, the case of LDC for country $\mathrm{H}$ with GAC prevailing in equilibrium. When $z(t)<\bar{z}$, a higher $r(t)$ than $\theta$ induces country $\mathrm{H}$ to consume less now than in the future, so that $c(t)<\bar{c}$, as is predicted by GAC. This takes place when LAC in country $\mathrm{F}$ is dominant. In contrast, if country $\mathrm{H}$ is dominant, a larger portion of the adjustment burden should be owed by country F's consumption: a higher $r(t)$ results in a lower $c^{*}(t)$ than $\bar{c}^{*}$, leaving $c(t)$ larger than $\bar{c}$ in country $\mathrm{H}$, so that GDC prevails reflecting H's LDC.

The transition dynamics of net foreign assets also depends on the property of the world felicity function and international heterogeneity of habits. By 
linearizing (3) and substituting (15) and (17) into the result, we can obtain

$$
\hat{b}_{t}=\frac{1}{\alpha(\theta-\omega)}\left\{\omega+\alpha-\alpha \kappa\left(\Omega^{H}-\Omega^{F}\right) b_{0}\right\} \hat{z}_{t} .
$$

Therefore, whether net foreign assets are positively or negatively correlated with consumption habits depends on: (i) whether the world felicity function displays GDC or GAC (the signs of $\omega+\alpha$ ); (ii) whether country $\mathrm{H}$ is more or less habituated to consumption than country $\mathrm{F}$ (the signs of $\Omega^{H}-\Omega^{F}$ ); and (iii) whether country $\mathrm{H}$ is initially a net creditor or debtor (the signs of $\left.b_{0}\right)$. Note that the last two factors capture the current-account effect of the interest-rate variation discussed in Property 4.

\subsection{Steady state}

From (1), (3), (4), (5), (6), (7), (8), (9), and (18), the steady state equilibrium, $\left(\bar{c}, \bar{c}^{*}, \bar{z}, \bar{z}^{*}, \bar{b}, \bar{b}^{*}, \bar{\lambda}, \bar{\xi}, \bar{r}\right)$, is determined by the following equations:

$$
\begin{aligned}
\bar{c} & =\bar{z} \\
\bar{\xi} & =\frac{1}{\theta+\alpha} u_{z}(\bar{c}, \bar{z}), \\
u_{c}(\bar{c}, \bar{z}) & =\bar{\lambda}-\alpha \bar{\xi}, \\
\bar{r} & =\theta, \\
\bar{r} \bar{b}+y & =\bar{c}+\tau, \\
\bar{b}-b_{0} & =\frac{1}{\alpha(\theta-\omega)}\left\{\omega+\alpha-\alpha \kappa\left(\Omega^{H}-\Omega^{F}\right) b_{0}\right\}\left(\bar{z}-z_{0}\right), \\
\bar{z}+\bar{z}^{*} & =Y, \\
\bar{c}+\bar{c}^{*} & =Y, \\
\bar{b}+\bar{b}^{*} & =0,
\end{aligned}
$$

where (19) represents $\dot{z}=0$ (see (1)); (20) represents $\dot{\xi}=0$ ((6)); (21) comes from the first order condition for consumption $c(4) ;(22)$ represents $\dot{\lambda}=0((5)) ;(23)$ represents $\dot{b}=0((3)) ;(24)$ comes from (18) evaluated at $t=0 ;(25)$ comes from (9); (26) and (27) represent the world market clearing conditions for the good and bonds $((7)$ and $(8))$.

By substituting (19) and (22) into (23), we obtain

$$
\theta \bar{b}+y=\bar{z}+\tau,
$$


which, together with (24), determines $(\bar{b}, \bar{z})$. Given this, $\bar{c}$ is given by (19); $\bar{\xi}$ by $(20) ; \bar{\lambda}$ by $(21) ; \bar{z}^{*}$ by $(25) ; \bar{c}^{*}$ by $(26)$; and $\bar{b}^{*}$ by $(27)$. Figure 2 depicts the determination of $(\bar{b}, \bar{z})$ : schedule $B B^{\prime}$ depicted with a positive slope represents (28), whereas schedule $S S^{\prime}$ represents (24).

\section{The Transfer Paradox}

\subsection{The steady-state effect of income transfer}

Suppose that country $\mathrm{H}$ is a creditor: $b_{0}>0$. Let us consider a permanent income transfer from the creditor $\mathrm{H}$ to the debtor $\mathrm{F}: d \tau>0 .{ }^{8}$ From (19), (24) and (28), we can obtain

$$
\begin{aligned}
& \frac{\mathrm{d} \bar{z}}{\mathrm{~d} \tau}=\frac{\mathrm{d} \bar{c}}{\mathrm{~d} \tau}=\left\{\omega(\alpha+\theta)-\alpha \theta \kappa\left(\Omega^{H}-\Omega^{F}\right) b_{0}\right\}^{-1} \alpha(\theta-\omega), \\
& \frac{\mathrm{d} \bar{b}}{\mathrm{~d} \tau}=\frac{1}{\alpha(\theta-\omega)}\left\{\omega+\alpha-\alpha \kappa\left(\Omega^{H}-\Omega^{F}\right) b_{0}\right\} \frac{\mathrm{d} \bar{z}}{\mathrm{~d} \tau},
\end{aligned}
$$

where it is assumed that $\omega(\alpha+\theta)-\alpha \theta \kappa\left(\Omega^{H}-\Omega^{F}\right) b_{0} \neq 0$ for the existence of the steady-state equilibrium.

The transfer payments have two effects on country $\mathrm{H}$ : a direct effect $\omega(\alpha+\theta)$ in $(29)$, due to a decrease in output income, and an indirect effect represented by $-\alpha \theta \kappa\left(\Omega^{H}-\Omega^{F}\right) b_{0}$, which is caused by changes in the interest rate. Steady state habits and consumption in country $\mathrm{H}$ are reduced by the transfer unless the indirect effect is positive and dominant. We cannot, however, rule out a priori the pathological possibility that the transfer payments increase the donor's habits and consumption due to increases in the interest rate. This causes the transfer paradox, as shown later. The effect on net foreign assets depends crucially on whether schedule $S S^{\prime}$ upward or downward sloping.

These adjustments can be illustrated by using Figure 3. An increase in $\tau$ shifts schedule $B B^{\prime}$ upward from $B_{0} B_{0}^{\prime}$ to $B_{1} B_{1}^{\prime}$, bringing the steady state point from $E_{0}$ to $E_{1}^{a}, E_{1}^{b}$, or $E_{1}^{c}$, corresponding to the $S S^{\prime}$ schedule $S_{a} S_{a}^{\prime}, S_{b} S_{b}^{\prime}$,

\footnotetext{
${ }^{8}$ Permanent transfer payments in the form of $\mathrm{d} \tau$, which are under consideration, is economically and analytically the same as once-and-for-all transfers $\mathrm{d} b_{0}$ of net foreign assets since international bond markets are perfect.
} 
or $S_{c} S_{c}^{\prime}{ }^{9}{ }^{9}$ Point $E_{1}^{c}$ represents the paradoxical case which we shall focus on next.

\subsection{The main result}

Let us now examine the welfare effects of the transfer. First, linearize the instantaneous utility $u$ around the steady state to obtain: $u\left(c_{t}, z_{t}\right)=$ $u(\bar{c}, \bar{z})+u_{c} \hat{c}_{t}+u_{z} \hat{z}_{t}$. Next, substituting (15) and $\hat{z}_{t}=\hat{z}_{0} \exp (\omega t)$ successively into this equation yields: $u\left(c_{t}, z_{t}\right)=u(\bar{z}, \bar{z})+\left\{\frac{u_{c}(\omega+\alpha)}{\alpha}+u_{z}\right\} \hat{z}_{0} \exp (\omega t)$. By substituting this into (2), the welfare at time zero is obtained as

$$
U_{0}=\frac{u(\bar{z}, \bar{z})}{\theta}+\frac{u_{c}(\omega+\alpha) / \alpha+u_{z}}{\theta-\omega}\left(z_{0}-\bar{z}\right) .
$$

From (29), the effect of transfer $\mathrm{d} \tau(>0)$ on donor H's welfare is thus given by

$$
\begin{aligned}
\frac{\mathrm{d} U_{0}}{\mathrm{~d} \tau} & =-\frac{\omega\left\{\left(1+\frac{\theta}{\alpha}\right) u_{c}+u_{z}\right\}}{\theta(\theta-\omega)} \frac{\mathrm{d} \bar{z}}{\mathrm{~d} \tau} \\
& =-\frac{(\alpha+\theta)(\alpha+\theta-\omega)}{\alpha \theta(2 \alpha+\theta)(1-\epsilon) u_{c c}}\left\{\frac{(\alpha+\theta) \omega}{\alpha \theta \kappa}-\left(\Omega^{H}-\Omega^{F}\right) b_{0}\right\}^{-1}
\end{aligned}
$$

whereas, from (9), the effect on recipient F's welfare is:

$$
\begin{aligned}
\frac{\mathrm{d} U_{0}^{*}}{\mathrm{~d} \tau} & =\frac{\omega\left\{\left(1+\frac{\theta}{\alpha}\right) u_{c}^{*}+u_{z}^{*}\right\}}{\theta(\theta-\omega)} \frac{\mathrm{d} \bar{z}}{\mathrm{~d} \tau} \\
& =\frac{(\alpha+\theta)(\alpha+\theta-\omega)}{\sigma \alpha \theta(2 \alpha+\theta)(1-\epsilon) u_{c c}}\left\{\frac{(\alpha+\theta) \omega}{\alpha \theta \kappa}-\left(\Omega^{H}-\Omega^{F}\right) b_{0}\right\}^{-1} .
\end{aligned}
$$

From the regularity assumption (C3), the first equations of (30) and (31) imply that the sign of the welfare effect is the same as that of $\mathrm{d} \bar{z} / \mathrm{d} \tau$ for country $\mathrm{H}$ and opposite for country F. ${ }^{10}$

\footnotetext{
${ }^{9}$ The above analysis can be applied to other income shocks with constant aggregate income. For example, an increase in the subjective discount rate $\theta$ shifts the $B B^{\prime}$ schedule upward or downward, depending on whether country $\mathrm{H}$ is initially a net debtor or creditor. Given the direction of the shift, the same analysis as in Figure 3 can be applied.
}

${ }^{10}$ Equations (30) and (31) imply:

$$
\frac{\mathrm{d} U_{0}}{\mathrm{~d} \tau}+\sigma \frac{\mathrm{d} U_{0}^{*}}{\mathrm{~d} \tau}=0
$$

meaning that the equilibrium dynamics we have obtained are indeed Pareto-efficient. 
From equations (30) and (31), the necessary and sufficient condition for the transfer paradox to occur is given as follows:

Proposition 2: Transfer payments $d \tau$ from country $H$ to $F$ immiserize the recipient and enrich the donor iff $(\alpha+\theta) \omega /(\alpha \theta \kappa)>\left(\Omega^{H}-\Omega^{F}\right) b_{0}$, i.e.,

$$
\frac{(\alpha+\theta)(\alpha+\theta-\omega)\left(u_{c c}+\sigma u_{c c}^{*}\right) \lambda^{*}}{\alpha \theta(2 \alpha+\theta) u_{c c} u_{c c}^{*}}>\left(\Omega^{H}-\Omega^{F}\right) b_{0} .
$$

Proposition 2 has the following four implications: First, since $b_{0}$ is positive by construction, the proposition implies that a necessary condition for the transfer paradox to take place is that $\Omega^{H}<\Omega^{F}$ : recipient country $\mathrm{F}$ is more habituated to consumption than donor country $\mathrm{H}: \omega^{F}>\omega^{H}$.

Second, the interest rate adjustment reflecting this habit heterogeneity induces the intertemporal terms of trade effect, thereby causing the transfer paradox. To see this, obtain from equation (17) and (30)

$$
\frac{\mathrm{d} r(0)}{\mathrm{d} \tau}=-\left\{\frac{(\alpha+\theta) \omega}{\alpha \theta \kappa}-\left(\Omega^{H}-\Omega^{F}\right) b_{0}\right\}^{-1} \frac{(\theta-\omega)\left(\Omega^{H}-\Omega^{F}\right)}{\theta} .
$$

The transfer paradox thus takes place only when the interest rate instantaneously jumps up, and hence only when the intertemporal terms of trade for the recipient deteriorates discretely. ${ }^{11}$ More specifically, when $\Omega^{H}<\Omega^{F}$, we can easily show that whenever the interest rate $r(0)$ goes up, the disposable income $y+r b-\tau$ for the donor initially increases: $\frac{\mathrm{d}}{\mathrm{d} \tau}\left\{b_{0} r(0)-\tau\right\}>0 .{ }^{12}$ It follows that the transfer payments benefits the donor only when the percentage rate of the initial rise in the interest rate is higher than the percentage rate of the initial decrease in the total wealth $b_{0}-\tau / r^{*}$ :

$$
\frac{\mathrm{d} r(0)}{\bar{r}}>\frac{(\mathrm{d} \tau) / \bar{r}}{b_{0}}
$$

${ }^{11}$ Conversely, given that recipient country $\mathrm{F}$ is more habituated to consumption than donor country $\mathrm{H}$, the interest rate instantaneously jumps up only when the transfer paradox occurs.

${ }^{12}$ We have $\frac{b_{0} \mathrm{~d} r(0)}{\mathrm{d} \tau}-1>0$ if and only if

$$
\omega(\alpha+\theta)>\alpha \kappa b_{0} \min \left\{\theta\left(\Omega^{H}-\Omega^{F}\right), \omega\left(\Omega^{H}-\Omega^{F}\right)\right\} .
$$

Given that $\Omega^{H}<\Omega^{F}$, this is equivalent to (32). 
This situation is somewhat similar to the "debt-relief Laffer curve" effect discussed by Krugman (1989): by using a tentative model, he points out the possibility that debt forgiveness actually increases expected payment on the wrong side of the "debt-relief Laffer curve". The discussion above shows that this kind of the Laffer-curve effect can be consistent with consumers' dynamic utility maximizing behavior. ${ }^{13}$

Third, roughly speaking, given that recipient $\mathrm{F}$ is more habituated to consumption than $\mathrm{H}$, the larger the initial net foreign assets $b_{0}$ held by donor $\mathrm{H}$, the more likely the paradox takes place. This is because a large $b_{0}$ exaggerates the perverse terms-of-trade effect. This statement is too rough, however, since an (exogenous) increase in $b_{0}$ may well affect the initial equilibrium resource allocation and hence the other parts of (32). A precise implication of $b_{0}$ is as follows. Let $I_{0}^{(*)}$ denote the initial steady-state GNP: $I_{0}^{(*)}=\theta b_{0}^{(*)}+y^{(*)}$, where note that $I_{0}+I_{0}^{*}=Y$ and $I_{0}^{(*)}=z_{0}^{(*)}$. Suppose now that

$$
\frac{(\alpha+\theta)(\alpha+\theta-\omega)\left(u_{c c}+\sigma u_{c c}^{*}\right) \lambda^{*}}{\alpha \theta(2 \alpha+\theta) u_{c c} u_{c c}^{*}}>\left(\Omega^{H}-\Omega^{F}\right) \frac{z_{0}}{\theta} .
$$

Then, for given $\left(I_{0}, I_{0}^{*}\right)$, and hence for given $\left(z_{0}, z_{0}^{*}\right)$, the larger $b_{0}$, the more likely the transfer paradox takes place. ${ }^{14}$

Fourth, (32) depends on the marginal felicities $u_{i}$ and $u_{i}^{*}(i=c, z)$ only through $\lambda^{*}\left(=u_{c}^{*}+\frac{\alpha}{\alpha+\theta} u_{z}^{*}\right)$ and $\sigma\left(=\frac{\lambda}{\lambda^{*}}=\left(u_{c}+\frac{\alpha}{\alpha+\theta} u_{z}\right) /\left(u_{c}^{*}+\frac{\alpha}{\alpha+\theta} u_{z}^{*}\right)\right)$. Note here that, as seen from the Euler equation (41) in Appendix B, $-\lambda^{(*)} /\left(c^{(*)} u_{c c}^{(*)}\right)$ represent the elasticities of intertemporal substitution. Condition (32) thus implies the following: Suppose that recipient $\mathrm{F}$ is more habituated to consumption than donor $\mathrm{H}$. Then, for given $\left(u_{i j}, u_{i j}^{*}, \alpha, \theta, \sigma, b_{0}\right)(i, j=c, z)$, the smaller $\lambda$ and $\lambda^{*}$, and hence the smaller the elasticities of intertemporal substitution, the more likely the paradox occurs. This is natural again since small elasticities of intertemporal substitution enlarge a response in the interest rate. ${ }^{15}$

\footnotetext{
${ }^{13}$ In Krugman (1989), the "debt-relief Laffer curve" effect takes place when debt forgiveness affects the probability distribution of the nature in favor of the "good" state. The effect is thus Pareto-improving: it is also beneficial to the debtor. In the present model, in contrast, equilibrium is always Pareto efficient, so that the "debt-relief Laffer curve" effect is definitely harmful to the debtor.

${ }^{14}$ Inequality (33) is necessary because $b_{0}$ cannot exceed $z_{0} / \theta$.

${ }^{15}$ From the same discussion, we can choose a set of parameter sets such that (32) holds by choosing the first derivatives $\left(u_{c},-u_{z}, u_{c}^{*},-u_{z}^{*}\right)$ arbitrarily small, with keeping $\sigma$ and
} 
All these discussions can be summarized as follows:

\section{Corollary:}

1. The transfer paradox takes place only when recipient country $F$ is more habituated to consumption than donor country $H$.

2. The transfer paradox takes place only when the interest rate initially jumps up, thereby increasing the total wealth of the donor.

3. Suppose that recipient $F$ is more habituated to consumption than donor $H$. Then, the possibility of the paradox can be characterized as follows:

(a) For given GNPs $\left(I_{0}, I_{0}^{*}\right)$ of the two countries, the larger the net foreign assets $b_{0}$ held by the donor, the more likely the transfer paradox occurs if (33) is met.

(b) For given $\left(u_{i j}, u_{i j}^{*}, \alpha, \theta, \sigma, b_{0}\right)(i, j=c, z)$, the smaller the elasticities of intertemporal substitution, the more likely the paradox occurs.

\subsection{The trick: relation to Samuelson}

A striking aspect of Proposition 2 is that it appears to be inconsistent with Samuelson's fundamental result: we have shown that the transfer paradox can occur even in a locally-stable, two-country equilibrium without any distortion.

The trick lies in a difference in the stability concept: The fundamental theorem requires the Walrasian stability, i.e., stability with respect to the Walrasian adjustment process, whereas the dynamics of our model are stable in the usual sense that the relevant characteristic root $\omega$, which characterizes the equilibrium dynamics of the economy, is negative.

To see this, let us consider an ad hoc Walrasian adjustment process for the time-zero international bond market as follows. Solve equation (24) for $b_{0}$ and eliminate $\bar{b}$ and $\bar{z}$ from the resulting equation by using (28) and (17), respectively, where (17) is evaluated at $t=+0$. The resultant equation, where $b_{0}$ is expressed as a function of the time-zero interest rate $r_{0}$, could be the other parameters constant. This point is discussed more explicitly in Section 5.2. 
regarded as a demand function for $b_{0}$. Letting $D\left(r_{0}\right)$ represent the demand function, it is given by

$$
D\left(r_{0}\right) \equiv \frac{\omega(\theta+\alpha)-\alpha \theta \kappa\left(\Omega^{H}-\Omega^{F}\right) b_{0}}{\alpha \theta \kappa\left(\Omega^{H}-\Omega^{F}\right)(\theta-\omega)}\left(r_{0}-\theta\right)-\frac{y-z_{0}-\tau}{\theta} .
$$

As in the usual rational-expectation models, we have so far implicitly assumed that the international bond market is instantaneously cleared by $r_{0}$ such that the demand $D\left(r_{0}\right)$ equals the existing stock $b_{0}$. Instead, imagine for exposition that the market can be cleared only "sluggishly" according to:

$$
\frac{\mathrm{d} r_{0}(s)}{\mathrm{d} s}=k\left(b_{0}-D\left(r_{0}(s)\right)\right),
$$

where $s$ represents some imaginary time; and $k(>0)$ is the adjustment speed. Equation (34) represents an imaginary Walrasian adjustment process where the interest rate rises "sluggishly" depending on the excess supply of the bond stock. With this process, the market could be said to be Walras-stable iff

$$
\left(D^{\prime}\left(r_{0}\right)=\right) \frac{\omega(\theta+\alpha)-\alpha \theta \kappa\left(\Omega^{H}-\Omega^{F}\right) b_{0}}{\alpha \theta \kappa\left(\Omega^{H}-\Omega^{F}\right)(\theta-\omega)}>0 .
$$

From this observation, we can show that the Walrasian instability is a necessary condition for the transfer paradox to occur:

Proposition 3: The transfer paradox takes place only if the international bond market at time zero is Walras-unstable with respect to the ad hoc Walrasian adjustment process given by (34).

Proof. Suppose that inequality (32) holds true. This implies (i) the numerator of (35) is positive; and (ii) $\Omega^{H}-\Omega^{F}<0$, which together imply $D^{\prime}\left(r_{0}\right)<0$.

We do not put Proposition 3 to show that the transfer paradox is unlikely to occur, but to show the consistency of our result to Samuelson's. Unlike in his (and other trade theorists') intrinsically-static models, however, we incorporate dynamics in a natural way by considering consumers' optimal saving behavior, showing that the steady state equilibria are locally saddlepoint stable. Given the modelling, stability should reasonably be discussed 
from the viewpoint of the equilibrium dynamics, as proposed by Burmeister and Long (1977). The local stability implies that the transfer paradox generically occurs at the neighborhoods of the corresponding steady state point. Epstein (1987) and Haaparanta (1989) also found that the transfer paradox is compatible with saddle-point stability of equilibrium dynamics. ${ }^{16}$ All these results imply that, given that the economy is always in a dynamic rational-expectation equilibrium, the transfer paradox cannot be ruled out by the stability argument. ${ }^{17}$

\section{Example: The case of quadratic felicity functions}

\subsection{Quadratic felicity functions}

To discuss more explicitly the possibilities of the transfer paradox, let us construct a simple example by using quadratic felicity functions, as in Becker and Murphy (1988) and Obstfeld (1992):

$$
u^{(*)}\left(c^{(*)}, z^{(*)}\right)=\phi_{c}^{(*)} c^{(*)}-\phi_{z}^{(*)} z^{(*)}+\phi_{c z}^{(*)} c^{(*)} z^{(*)}-\phi_{c c}^{(*)} \frac{c^{(*) 2}}{2}-\phi_{z z}^{(*)} \frac{z^{(*) 2}}{2}
$$

for which case the partial derivatives of $u^{(*)}\left(c^{(*)}, z^{(*)}\right)$ evaluated at the initial steady state are given by

$$
\begin{aligned}
& u_{c}^{(*)}=\phi_{c}^{(*)}-\left(\phi_{c c}^{(*)}-\phi_{c z}^{(*)}\right) z_{0}^{(*)}, \\
& u_{z}^{(*)}=-\left\{\phi_{z}^{(*)}-\left(\phi_{c z}^{(*)}-\phi_{z z}^{(*)}\right) z_{0}^{(*)}\right\}, \\
& u_{c c}^{(*)}=-\phi_{c c}^{(*)}, u_{c z}^{(*)}=\phi_{c z}^{(*)}, u_{z z}^{(*)}=-\phi_{z z}^{(*)} .
\end{aligned}
$$

For the regularity conditions to be satisfied, we restrict the values of parameters $\phi_{i}^{(*)}$ and $\phi_{i j}^{(*)}$ as follows:

$$
\text { 1. } \phi_{c}^{(*)}>0, \phi_{z}^{(*)}>0, \phi_{c c}^{(*)}>0, \phi_{z z}^{(*)}>0, \phi_{c z}^{(*)} \gtreqless 0 ;
$$

${ }^{16}$ However, Epstein (1987) and Haaparanta (1989) do not elucidate how their results can be consistent with Samuelson's finding. We can show that Epstein's transfer paradox also takes place only when the international capital market is unstable with respect to an adhoc Walrasian adjustment process.

${ }^{17}$ We do not intend to say that any "disequilibrium" dynamics are meaningless. Our point is that the stability arguments based on ad hoc adjustment processes such as the Walrasian process could not be a strong reason for ruling out a priori the possibilities of the transfer paradox or other phenomena derived from rational agent models. 
2. $\phi_{c}^{(*)}-\left(\phi_{c c}^{(*)}-\phi_{c z}^{(*)}\right) z_{0}^{(*)}>0, \phi_{z}^{(*)}-\left(\phi_{c z}^{(*)}-\phi_{z z}^{(*)}\right) z_{0}^{(*)}>0$ for $u_{c}^{(*)}>0$ and $u_{z}^{(*)}<0$

3. $\phi_{c}^{(*)}-\left(\phi_{c c}^{(*)}-\phi_{c z}^{(*)}\right) z_{0}^{(*)}-\left\{\phi_{z}^{(*)}-\left(\phi_{c z}^{(*)}-\phi_{z z}^{(*)}\right) z_{0}^{(*)}\right\}=\phi_{c}^{(*)}-\phi_{z}^{(*)}-$ $\left(\phi_{c c}^{(*)}-2 \phi_{c z}^{(*)}+\phi_{z z}^{(*)}\right) z_{0}^{(*)}>0$ for $u_{c}^{(*)}+u_{z}^{(*)}>0 ;$ and

4. $\phi_{c c}^{(*)} \phi_{z z}^{(*)}-\phi_{c z}^{(*) 2}>0$ for concavity.

\subsection{Possibilities of the transfer paradox}

By using the felicity functions, we shall show that there actually exist parameter values that allow the transfer paradox. With felicity functions (36), the necessary and sufficient condition (32) for the paradox to occur reduces to

$$
-\frac{(\alpha+\theta)(\alpha+\theta-\omega)}{\alpha \theta(2 \alpha+\theta)} \frac{\phi_{c c}+\sigma \phi_{c c}^{*}}{\phi_{c c} \phi_{c c}^{*}} \lambda^{*}>\left(\Omega^{H}-\Omega^{F}\right) b_{0},
$$

where

$$
\begin{aligned}
& \lambda^{*}\left(\equiv u_{c}^{*}+\frac{\alpha}{\theta+\alpha} u_{z}^{*}\right)=\phi_{c}^{*}-\left(\phi_{c c}^{*}-\phi_{c z}^{*}\right) z_{0}^{*}-\frac{\alpha}{\alpha+\theta}\left\{\phi_{z}^{*}-\left(\phi_{c z}^{*}-\phi_{z z}^{*}\right) z_{0}^{*}\right\} \\
& \sigma\left(\equiv \frac{\lambda}{\lambda^{*}}\right)=\frac{\phi_{c}-\left(\phi_{c c}-\phi_{c z}\right) z_{0}-\frac{\alpha}{\alpha+\theta}\left\{\phi_{z}-\left(\phi_{c z}-\phi_{z z}\right) z_{0}\right\}}{\phi_{c}^{*}-\left(\phi_{c c}^{*}-\phi_{c z}^{*}\right) z_{0}^{*}-\frac{\alpha}{\alpha+\theta}\left\{\phi_{z}^{*}-\left(\phi_{c z}^{*}-\phi_{z z}^{*}\right) z_{0}^{*}\right\}} \\
& \Omega^{H}=\left(\phi_{c z}-\frac{\alpha}{\theta+2 \alpha} \phi_{z z}\right) / \phi_{c c} ; \Omega^{F}=\left(\phi_{c z}^{*}-\frac{\alpha}{\theta+2 \alpha} \phi_{z z}^{*}\right) / \phi_{c c}^{*}
\end{aligned}
$$

Start with choosing parameter values satisfying the regularity conditions, such that:

- (a) country $\mathrm{F}$ is more habituated to consumption than $\mathrm{H}: \Omega^{F}>\Omega^{H}$;

- (b) country $\mathrm{H}$ is initially a creditor: $b_{0}>0$;

- $(\mathrm{c}) \phi_{c c}^{(*)}-\phi_{c z}^{(*)}>0, \phi_{c z}^{(*)}-\phi_{z z}^{(*)}>0$. 
From two properties (a) and (b), the right hand side of inequality (37) is negative. Since our purpose in this subsection is to verify the possibility of the paradox, we should start with some parameter set under which the right hand side of inequality (37) is larger than the left hand side.

But note that, by choosing $\left(\phi_{c}, \phi_{z}, \phi_{c}^{*}, \phi_{z}^{*}\right)$ appropriately, we can make the values of $u_{c}^{(*)}$ and $u_{z}^{(*)}$ and hence $\lambda^{*}$ arbitrarily close to zero, with fixing the value of $\sigma$ and other components of (37) at the values initially chosen. That is, we can take limits of $\lambda^{*} \downarrow+0$ by taking limits of $\left(u_{c},-u_{z}, u_{c}^{*},-u_{z}^{*}\right) \downarrow+0$, which, in turn, can be taken, with keeping $\sigma$ constant, by considering the limit:

$$
\left(\phi_{c}, \phi_{z}, \phi_{c}^{*}, \phi_{z}^{*}\right) \downarrow\left(\left(\phi_{c c}-\phi_{c z}\right) z_{0},\left(\phi_{c z}-\phi_{z z}\right) z_{0},\left(\phi_{c c}^{*}-\phi_{c z}^{*}\right) z_{0}^{*},\left(\phi_{c z}^{*}-\phi_{z z}^{*}\right) z_{0}^{*}\right),
$$

which is positive from condition (c). For any $\left(\alpha, \theta, \phi_{c c}^{(*)}, \phi_{c z}^{(*)}, \phi_{z z}^{(*)}, z_{0}, z_{0}^{*}, b_{0}\right)$ that satisfies the regularity conditions and conditions (a) though (c) above, this means that there always exists a continuum of the values for $\left(\phi_{c}, \phi_{z}, \phi_{c}^{*}, \phi_{z}^{*}\right)$ and hence for $\lambda^{*}$ that validates inequality (37).

For example, limit (39) proposed above can be conducted as follows. First, for a given regular value of $\left(\alpha, \theta, \phi_{c c}^{(*)}, \phi_{c z}^{(*)}, \phi_{z z}^{(*)}, z_{0}, z_{0}^{*}, b_{0}\right)$, construct $\left(\phi_{c}, \phi_{z}, \phi_{c}^{*}, \phi_{z}^{*}\right)$ as

$$
\begin{aligned}
\phi_{c} & =\left(\phi_{c c}-\phi_{c z}\right) z_{0}+\varepsilon_{c}, \\
\phi_{z} & =\left(\phi_{c z}-\phi_{z z}\right) z_{0}+\varepsilon_{z}, \\
\phi_{c}^{*} & =\left(\phi_{c c}^{*}-\phi_{c z}^{*}\right) z_{0}^{*}+\varepsilon_{c}^{*}, \\
\phi_{z}^{*} & =\left(\phi_{c z}^{*}-\phi_{z z}^{*}\right) z_{0}^{*}+\varepsilon_{z}^{*},
\end{aligned}
$$

where the $\varepsilon_{i}^{(*)}$ 's are positive parameters. Let $k$ and $n$ be constant parameters such that $0<k<\frac{\alpha+\theta}{\alpha}$ and $n>0$. Then, by setting the $\varepsilon_{i}^{(*)}$ 's as

$$
\varepsilon_{z}=k \varepsilon_{c}, \varepsilon_{c}^{*}=\frac{1}{n} \varepsilon_{c}, \text { and } \varepsilon_{z}^{*}=\frac{k}{n} \varepsilon_{c}
$$

we obtain from (38)

$$
\sigma=n,
$$

which is a positive constant by construction, irrespective of the $\varepsilon_{i}^{(*)}$ 's values. From (40), by taking limit $\varepsilon_{c} \rightarrow 0$, we can thus take limit (39) and hence $\lambda^{*} \downarrow$ +0 , with keeping $\sigma$ and other components appearing in (37) constant. (Note: 
Components in (37) other than $\left(\lambda^{*}, \sigma\right)$ do not depend on $\left(\phi_{c}, \phi_{z}, \phi_{c}^{*}, \phi_{z}^{*}\right)$ and hence on the $\varepsilon_{i}^{(*)}$ 's.) In this way, by choosing an arbitrarily small $\varepsilon_{c}$, we make the absolute value of the left hand side of (37) arbitrarily small, with keeping the right hand side constant.

\subsection{Numerical examples}

In view of (37), let us now define the paradox indicator function as:

$$
\begin{aligned}
& T P X\left(\alpha, \theta, \phi_{c}, \phi_{z}, \phi_{c c}, \phi_{c z}, \phi_{z z}, \phi_{c}^{*}, \phi_{z}^{*}, \phi_{c c}^{*}, \phi_{c z}^{*}, \phi_{z z}^{*}, z_{0}, z_{0}^{*}, b_{0}\right) \\
= & -\frac{(\alpha+\theta)(\alpha+\theta-\omega)}{\alpha \theta(2 \alpha+\theta)} \frac{\phi_{c c}+\sigma \phi_{c c}^{*}}{\phi_{c c} \phi_{c c}^{*}} \lambda^{*}-\left(\Omega_{H}-\Omega_{F}\right) b_{0} \\
& \left\{\begin{array}{l}
>0 \text { for the paradox case }, \\
\leq 0 \text { for the normal case. }
\end{array}\right.
\end{aligned}
$$

For the reference case, we consider the parameter set listed in Table 1 which satisfies all the regularity conditions and conditions (a)-(c):

\begin{tabular}{|c|c|c|c|c|c|c|c|c|c|c|c|c|c|c|}
\hline$\alpha$ & $\theta$ & $\phi_{c}$ & $\phi_{z}$ & $\phi_{c c}$ & $\phi_{c z}$ & $\phi_{z z}$ & $\phi_{c}^{*}$ & $\phi_{z}^{*}$ & $\phi_{c c}^{*}$ & $\phi_{c z}^{*}$ & $\phi_{z z}^{*}$ & $z_{0}$ & $z_{0}^{*}$ & $b_{0}$ \\
\hline \hline 1 & 1 & 15.2 & 0.1 & 16 & 1 & 1 & 8.7 & 0.6 & 10 & 1.5 & 1 & 1 & 1 & 0.4 \\
\hline
\end{tabular}

Table 1: Reference Parameter Values

With these parameter values, the following properties are valid:

1. We have $\Omega^{H}=4.1667 \times 10^{-2}$ and $\Omega^{F}=0.11667$, implying that $\Omega^{F}>$ $\Omega^{H}>0$, and hence $\omega^{F}>\omega^{H}$, that is country $\mathrm{F}$ is more habituated to consumption. This is a necessary condition for the transfer paradox to occur.

2. The stable root amounts to: $\omega=-0.92775$, so that GAC prevails since $\omega+\alpha>0$.

3. $\lambda=\lambda^{*}=0.15$ and the weight applied to country F's felicity in constructing function $v$ is also unity: $\sigma=1$.

4. However, the transfer paradox does not take place in the reference case since $T P X=-1.7576 \times 10^{-2}<0$. 
As shown in Corollary, the transfer paradox likely occurs under a large $b_{0}$. We can obtain the critical value $\beta$ for $b_{0}$ required for the transfer paradox to occur under the reference case by solving:

$$
\operatorname{TPX}(1,1,15.2,0.1,16,1,1,8.7,0.6,10,1.5,1,1,1, \beta)=0 .
$$

This can be computed to obtain $\beta \simeq 0.64$, which is smaller than $z_{0} / \theta=1$. Therefore, if $b_{0}$ is larger 0.64 , transfer payments immiserize the recipient, insofar as $b_{0}<1$. In fact, for example, when $b_{0}=0.7, T P X$ amounts to $4.92 \times 10^{-3}$, implying the paradox.

Let us next check item 3(b) in Corollary by examining the effect of changes in $\left(\phi_{c}^{*}, \phi_{z}^{*}\right)$. Under the reference values for the other parameters, $\left(\phi_{c}^{*}, \phi_{z}^{*}\right)$ should satisfy $\phi_{c}^{*}>8.5 ; \phi_{z}^{*}>0.5 ; \phi_{c}^{*}-\phi_{z}^{*}>8.0$ to meet the regularity conditions. A small $\phi_{c}^{*}-\phi_{z}^{*}-8.0$ implies a small $\lambda^{*}$ and hence a small elasticity of intertemporal substitution of country $\mathrm{F}$, where the elasticity of intertemporal substitution is given by $\lambda^{*} / \phi_{c c}^{*}=\lambda^{*} / 10$ in the reference case. Table 2 summarizes the relationship among $\left(\phi_{c}^{*}, \phi_{z}^{*}\right)$, elasticity of intertemporal substitution (EIS) $\lambda^{*} / \phi_{c c}^{*}$, and $\beta$. In the reference case $\left(\phi_{c}^{*}, \phi_{z}^{*}\right)=(8.7,0.6)$, EIS amounts to 0.015 , and a $b_{0}$ larger than 0.64 results in the paradox. As is expected, in case 1, in which EIS is much smaller than in the reference case, the paradox can occur for a smaller $b_{0}(\beta=0.25)$. In contrast, in case 2 , EIS equals 0.028 , so that the paradox unlikely takes place since $\beta$ is almost equal to one. In case 3, EIS is too large for the paradox to occur. ${ }^{18}$

\begin{tabular}{|c||c|c|c|}
\hline & $\left(\phi_{c}^{*}, \phi_{z}^{*}\right)$ & EIS $\left(\lambda^{*} / \phi_{c c}^{*}\right)$ & $b_{0}$ for the paradox $(\beta)$ \\
\hline \hline reference case & $(8.7,0.6)$ & 0.015 & 0.64 \\
\hline case 1 & $(8.51,0.509)$ & 0.0006 & 0.25 \\
\hline case 2 & $(8.93,0.8)$ & 0.028 & 0.98 \\
\hline case 3 & $(9,1)$ & 0.125 & $\emptyset$ \\
\hline
\end{tabular}

Table 2: Elasticity of Intertemporal Substitution, External Assets, and the Possibility of the Transfer Paradox.

${ }^{18}$ To be precise, these results are more than predicted by the analytical result $3(\mathrm{~b})$ in Corollary. When we compute the results in Table 2, all the effects on parameters $\sigma$ and $\omega$ are incoporated, whereas in statement 3(b) in Corollary, we fix these parameters. 


\section{Concluding remarks}

We have analyzed the transfer problem by using a one-good two-country model with infinitely-lived, heterogeneously habit-forming consumers. Due to pecuniary externalities through endogenous interest-rate adjustments, the equilibrium consumption-habit dynamics are characterized by a world felicity function constructed from the individual countries' felicity functions, which determines intertemporal consumption-complementarities prevailing in the two-country equilibrium. International transfer payments can harm the recipient and benefit the donor, depending on: (i) international heterogeneity in the preference for habits; (ii) initial asset distribution; and (iii) intertemporal consumption-complementarities in the world. In particular, when the debtor is more habituated to consumption than the creditor, transfer from the creditor to the debtor raises the interest rate in transition through changes in time preference. This intertemporal terms of trade effect causes the paradox.

Our result implies that the transfer paradox cannot be ruled out by the stability argument, given that the economy is always in the rationalexpectation dynamic equilibrium. We never intend to say that any "disequilibrium" analysis is meaningless; rather it must be examined whether the paradoxical result can still survive when stable adjustment processes in market disequilibrium are properly modeled from the micro-founded rational behavior of economic agents. It must be an important research topic in the future. 


\section{Appendices}

\section{A Proof of Property 1}

Note first that $\lambda / \lambda^{*}$ which is constant because $\dot{\lambda}_{t} / \lambda_{t}=\dot{\lambda}_{t}^{*} / \lambda_{t}^{*}$ from (5). By eliminating $c^{*}$ and $z^{*}$ using (7) and (9) from the foreign counterpart of (4), combining the resultant equation and (4) yields

$$
\frac{u_{c}(c, z)+\alpha \xi}{u_{c}^{*}(Y-c, Y-z)+\alpha \xi^{*}}=\frac{\lambda}{\lambda^{*}}=\text { constant. }
$$

By totally differentiating this, we can obtain

$$
\hat{c}=-\frac{\lambda^{*} u_{c z}+\lambda u_{c z}^{*}}{\lambda^{*} u_{c c}+\lambda u_{c c}^{*}} \hat{z}-\frac{\alpha \lambda^{*}}{\lambda^{*} u_{c c}+\lambda u_{c c}^{*}} \hat{\xi}+\frac{\alpha \lambda}{\lambda^{*} u_{c c}+\lambda u_{c c}^{*}} \hat{\xi}^{*} .
$$

Substitute this equation into (1), (6), and the foreign counterpart of (6) and eliminate $c^{*}$ and $z^{*}$ using (7) and (9) from the resulting equation. Then, we can derive the autonomous dynamic equation system with respect to $\left(\hat{z}, \hat{\xi}, \hat{\xi}^{*}\right)$ as

$$
\begin{aligned}
& \dot{z}=-\alpha\left(\frac{\lambda^{*} u_{c z}+\lambda u_{c z}^{*}}{\lambda^{*} u_{c c}+\lambda u_{c c}^{*}}+1\right) \hat{z}-\frac{\alpha^{2} \lambda^{*}}{\lambda^{*} u_{c c}+\lambda u_{c c}^{*}} \hat{\xi}+\frac{\alpha^{2} \lambda}{\lambda^{*} u_{c c}+\lambda u_{c c}^{*}} \hat{\xi}^{*}, \\
& \dot{\xi}=\left\{\frac{u_{c z}\left(\lambda^{*} u_{c z}+\lambda u_{c z}^{*}\right)}{\lambda^{*} u_{c c}+\lambda u_{c c}^{*}}-u_{z z}\right\} \hat{z}+\left(\theta+\alpha+\frac{\alpha u_{c z} \lambda^{*}}{\lambda^{*} u_{c c}+\lambda u_{c c}^{*}}\right) \hat{\xi}-\frac{\alpha u_{c z} \lambda}{\lambda^{*} u_{c c}+\lambda u_{c c}^{*}} \hat{\xi}^{*}, \\
& \dot{\xi}^{*}=-\left\{\frac{u_{c z}^{*}\left(\lambda^{*} u_{c z}+\lambda u_{c z}^{*}\right)}{\lambda^{*} u_{c c}+\lambda u_{c c}^{*}}-u_{z z}^{*}\right\} \hat{z}-\frac{\alpha u_{c z}^{*} \lambda^{*}}{\lambda^{*} u_{c c}+\lambda u_{c c}^{*}} \hat{\xi}+\left(\theta+\alpha+\frac{\alpha u_{c z}^{*} \lambda^{*}}{\lambda^{*} u_{c c}+\lambda u_{c c}^{*}}\right) \hat{\xi}^{*} .
\end{aligned}
$$

From the definitions (10) and (11) of $v$ and $\varsigma$, respectively, this autonomous system can be reduced to (12).

\section{B Derivation of (17)}

From (4), local consumption dynamics can be expressed using time preference

$$
\begin{gathered}
\rho \equiv-\left.\frac{\mathrm{d} \ln \left(u_{c}(c, z)+\alpha \xi\right)}{\mathrm{d} t}\right|_{\dot{c}=0} \text { as } \\
\dot{c}=-\frac{\lambda}{u_{c c}}(\hat{r}-\hat{\rho}),
\end{gathered}
$$


where

$$
\hat{\rho}=\frac{\alpha\left(u_{z z}+u_{c z}\right)}{\lambda} \hat{z}-\frac{\alpha(\theta+\alpha)}{\lambda} \hat{\xi}
$$

Since $\dot{c}=\omega \hat{c},(15)$ implies

$$
\dot{c}=\omega\left(\frac{\omega+\alpha}{\alpha}\right) \hat{z} \text {. }
$$

Substituting the last two equations into (41), we obtain:

$$
\hat{r}=\frac{-u_{c c} \omega(\omega+\alpha)+\alpha^{2}\left(u_{z z}+u_{c z}\right)}{\alpha \lambda} \hat{z}-\frac{\alpha(\theta+\alpha)}{\lambda} \hat{\xi}
$$

To eliminate $\hat{\xi}$ from the above, substitute $\dot{\xi}=\omega \hat{\xi}$ and (15) into (6) to obtain

$$
\hat{\xi}=\frac{(\omega+\alpha) u_{c z}+\alpha u_{z z}}{\alpha(\theta+\alpha-\omega)} \hat{z} .
$$

By substituting this equation into (42), we can obtain (17). 


\section{References}

Becker, G. and K. Murphy, 1988, A theory of rational addiction, Journal of Political Economy 96, 675-700.

Bhagwati, J.N., R.A. Brecher, and T. Hatta, 1983, The generalized theory of transfers and welfare: Bilateral transfers in a multilateral world, American Economic Review 73, 606-618.

Bhagwati, J.N., R.A. Brecher, and T. Hatta, 1985, The generalized theory of transfers and welfare: Exogenous (policy-imposed) and endogenous (transfer-induced) distortions, Quarterly Journal of Economics 100, 697-714.

Boldrin, M., L.J. Christiano, and J.D.M. Fisher, 2001, Habit persistence, asset returns, and the business cycle, American Economic Review $91,149-166$.

Braun, P., G. Constantinides, and W. Ferson, 1993, Time nonseparability in aggregate consumption: International evidence, European Economic Review 37, 897-920.

Brecher, R.A. and J.N. Bhagwati, 1982, Immiserizing transfers from abroad, Journal of International Economics 13, 353-364.

Burmeister, E. and Ngo Van Long, 1977, On some unresolved questions in capital theory: An application of Samuelson's correspondence principle, Quarterly Journal of Economics 91, 289-314.

Campbell, J. Y. and J. H. Cochrane, 1999, By force of habit: A consumption-based explanation of aggregate stock market behavior, Journal of Political Economy 107, 205-251.

Carrol, C. D., J. Overland, and D. N. Weil, 2000, Saving and growth with habit formation, American Economic Review 90, 341-355.

Djajić, S., S. Lahiri, and P. Raimondos-Møller, 1998, The transfer problem and the intertemporal terms of trade, Canadian Journal of Economics $31,427-436$.

Djajić, S., S. Lahiri, and P. Raimondos-Møller, 2001, Logic of aid in an international setting, mimeo. 
Epstein, L.G., 1987, A simple dynamic general equilibrium model, Journal of Economic Theory 41, 68-95.

Gale, D., 1974, Exchange equilibrium and coalition: An example, Journal of Mathematical Economics 1, 63-66.

Galor, O. and H.M. Polemarchakis, 1987, Intertemporal equilibrium and the transfer paradox, Review of Economic Studies 54, 147-156.

Haaparanta, P., 1989, The intertemporal effects of international transfers, Journal of International Economics 26,371-382.

Ikeda, S. and I. Gombi, 1999, Habits, costly investment, and current account dynamics, Journal of International Economics 49, 363-384.

Ikeda, S. and I. Gombi, 2001, Global habit formation: A two-country analysis, mimeo.

Kemp, M., 1987, Correspondence principle, in: J. Eatwell, M. Milgate, and P. Newman eds., The New Palgrave: A Dictionary of Economics, London: Macmillan Press.

Krugman, P., 1989, Market-based debt-reduction schemes, in: J.A. Frenkel, M.P. Dooley, and P. Wickham eds., Analytical Issues in Debt, International Monetary Fund, 258-278.

Mansoorian, A., 1993, Habit persistence and the Harberger-LaursenMetzler effect in an infinite horizon model, Journal of International Economics 34, 153-166.

Naik, N. and M. Moore, 1996, Habit formation and intertemporal substitution in individual food consumption, Review of Economics and Statistics LXXVIII, 321-328.

Obstfeld, M., 1992, International adjustment with habit-forming consumption: A diagrammatic exposition, Review of International Economics $1,32-48$.

Ryder, H. E. and Heal, G. M., 1973, Optimal growth with intertemporally dependent preference, Review of Economic Studies 40, 1-33. 
Samuelson, P., 1947, Foundations of Economic Analysis, Cambridge: Harvard University Press.

Samuelson, P., 1952, The transfer problem and transport costs: The terms of trade when impediments are absent, Economic Journal 62, 278-304.

Samuelson, P., 1954, The transfer problem and transport costs, 2: Analysis of effects of trade impediments, Economic Journal 64, 264289.

Yano, M., 1983, Welfare aspects of the transfer problem, Journal of International Economics 15, 277-289.

Yano, M. and J.B. Nugent, 1999, Aid, nontraded goods, and transfer paradox in small countries, American Economic Review 89, 431-449. 


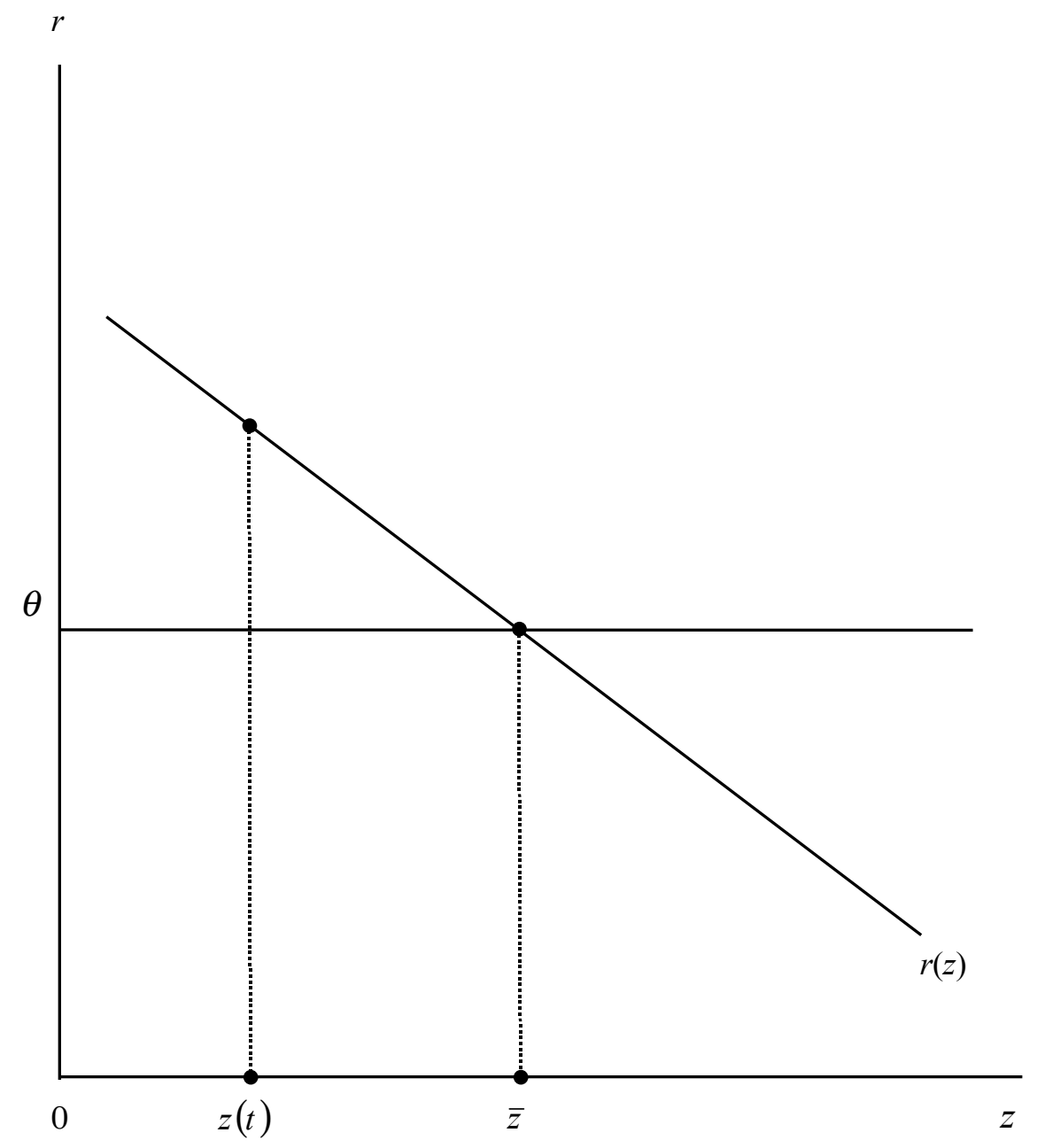

Figure 1. Equilibrium relationship between $r$ and $z$ :

The case of $\omega^{F}>\omega^{H}$. 


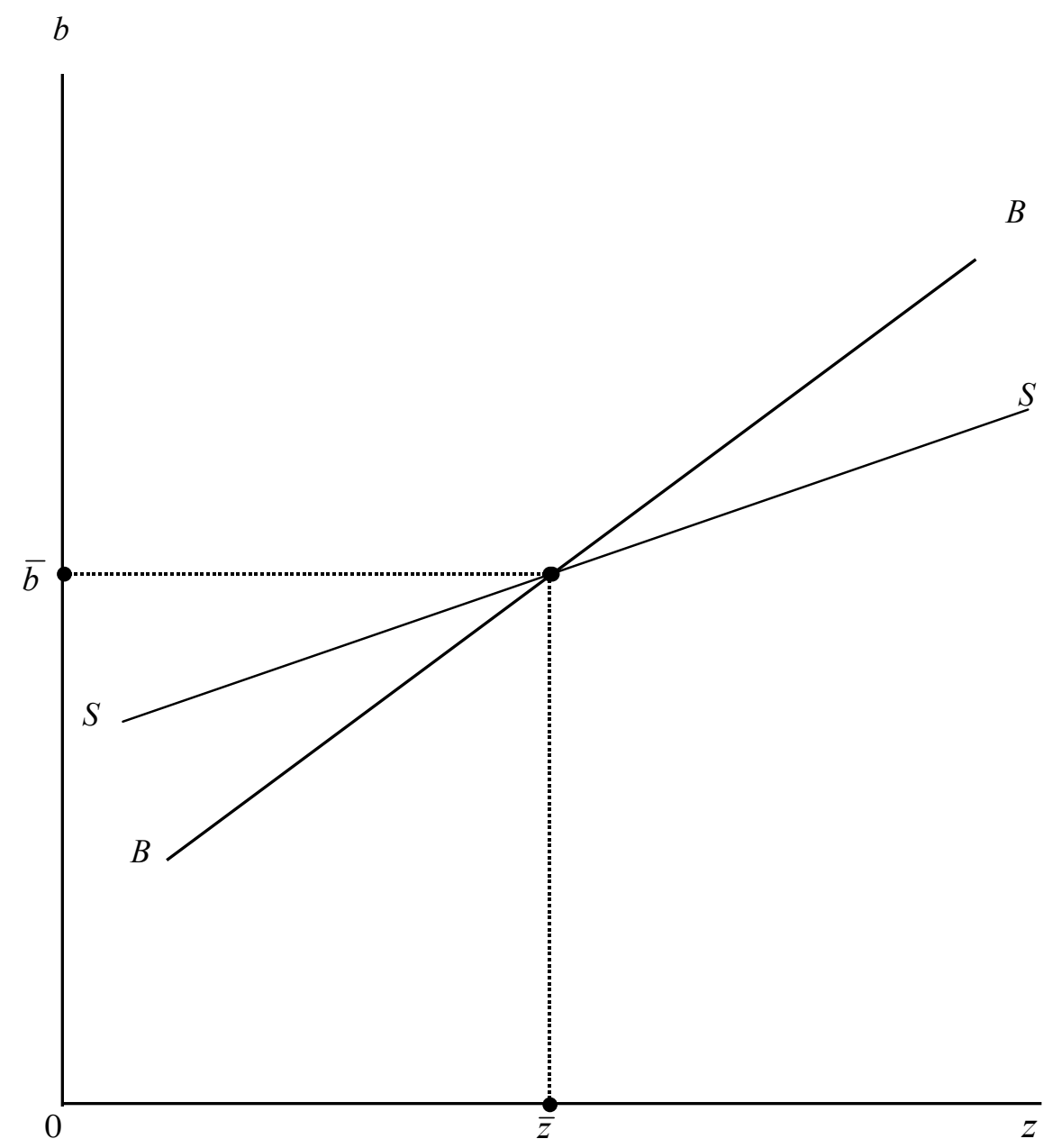

Figure 2. Steady state equilibrium:

The case of $\frac{1}{\theta}>\frac{\omega+\alpha-\alpha \kappa\left(\Omega^{H}-\Omega^{F}\right) b_{0}}{\alpha(\theta-\omega)}$. 


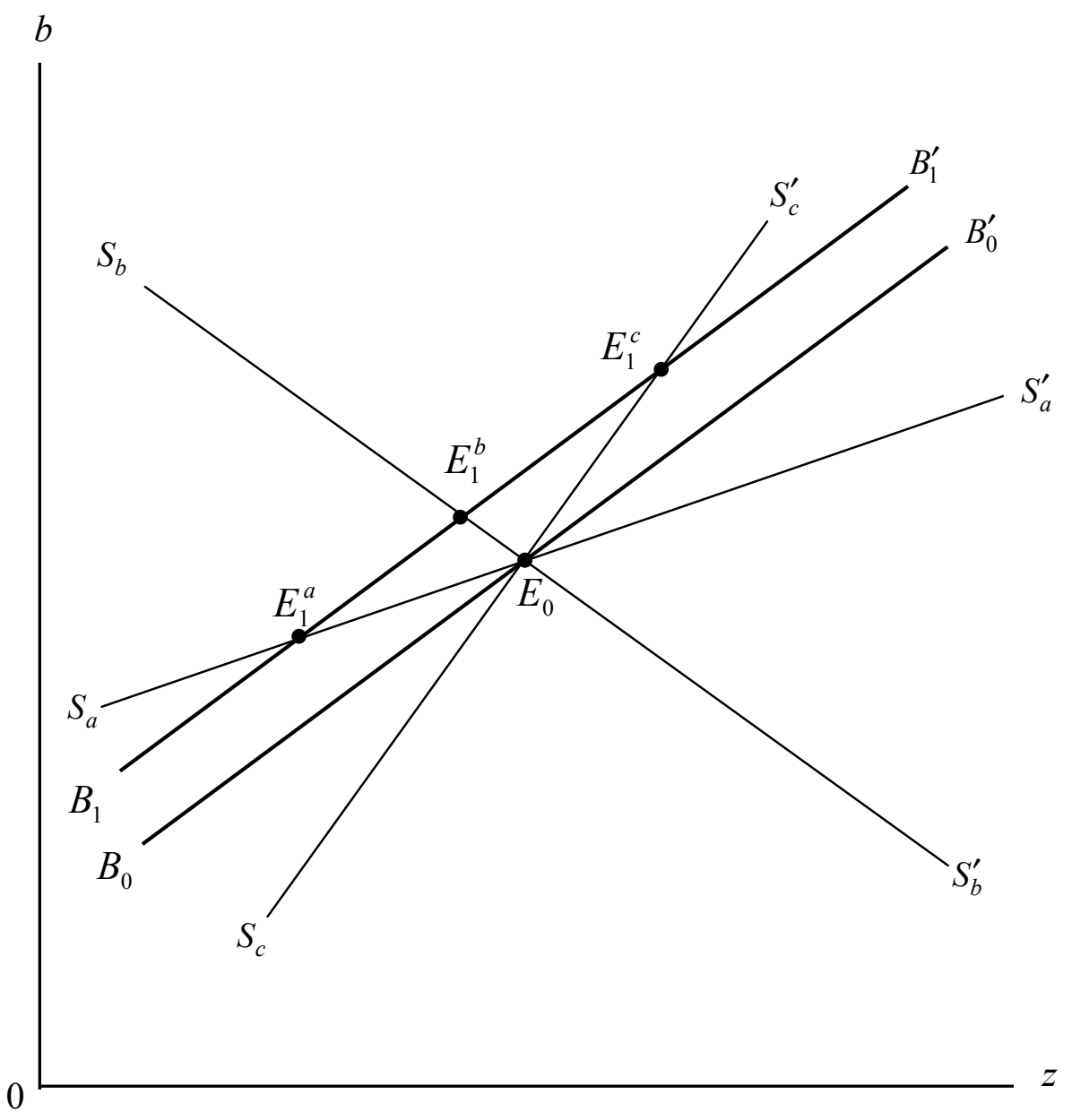

Figure 3. The effects of income transfers. 